\title{
Contemporary Cosmological Paradigms and their Impact on Educational Research
}

\author{
Serhii Horokhov \\ PhD, National Pedagogical Dragomanov University (Kyiv, Ukraine) \\ E-mail: s.v.horokhov@gmail.com \\ ORCID: 0000-0003-1772-5588
}

\section{Galina Zhukova}

Ph.D., Senior Lecturer, National University of Physical Education and Sports of Ukraine (Kyiv, Ukraine)

E-mail: galinazh1299@gmail.com

ORCID: 0000-0002-1248-4669

Three modern cosmological models are considered in the article: the Standard Cosmological Model, the theory "Evolving Matter" and the Mental Universe, which, according to the authors, clearly demonstrate the change of the worldview paradigm in modern society: a rational worldview gives way to an irrational world view, or a new form of idealism. In the article, the authors answer the question: how the change of cosmological paradigms affects the educational research. As a result of the study, the authors come to the conclusion that under the influence of the modern hypothesis of the Intelligent Matter and the Mental Universe rethinking of the subject and object of the educational research takes place. The subject of the educational research is the neural structure of the brain and the peculiarities of impact on it in ontogenesis, and the object of the educational research becomes the involvement of a neural structure in cosmic processes and the features of its development under the influence of the cosmos.

Keywords: cosmological paradigms, educational research, Mental Universe, Standard Cosmological Model, theory "Evolving Matter”, Intelligent Matter

Received: January 11, 2018; accepted: April 3, 2018

Philosophy and Cosmology, Volume 21, 2018: 14-20

DOI: $10.29202 /$ phil-cosm/21/2

\section{Introduction}

Considering the strategies of systematization of the theories of education in the history of culture, Oleg Bazaluk established that, in principle, the diversity of the theories of education in histories of culture can be divided according to the two lines of development:

(C) Horokhov, Serhii, 2018

(C) Zhukova, Galina, 2018 
Plato and Isocrates. The theories of education of Plato's line set the ideal of form building, answering the question: "Who should be educated in the younger generations?" The theories of education of Isocrates' line by daily educational practices ensure the achievement of the designated ideal, i.e. focus more on finding answers to the question "How to educate the younger generation?" [Bazaluk, 2017: 22-23].

One of the criteria according to which Bazaluk has systematized the educational research in the history of culture is the cosmological paradigm dominating in society. Since the time of Plato, it has been established that the features of human ideas about the Universe have a direct effect on the educational research. Let us consider briefly the contemporary cosmological paradigms and evaluate their impact on the educational research.

\section{The Standard Cosmological Model}

The Standard Cosmological Model, in particular a cosmological chaotic inflationary model, is the basic model in the modern cosmology. The first version of the inflationary theory was presented in 1981 by the American physicist and cosmologist Alan Guth Harvey. According to the inflationary theory, in the Universe, at different spatial and temporal intervals, spontaneous breakings occur. At each point where the breaking occurs, the Universe starts expanding, and new areas arise. Most of the time, the expansion is negligible. As the process is chaotic, at one point a bubble appeared, the expansion of which lasted long enough to create the Universe, structurally resembling our Universe. Expansion is eternal, big explosions happen constantly; some Universes branch off from other Universes. Under this scenario, these Universes can "blossom out" with other Universes, thus creating a "Multiverse". According to the theory of inflation, spontaneous breakages can occur anywhere, even in our Universe, meaning that, from our Universe another one could branch off. It also means that our Universe itself could also branch off from another Universe. According to the chaotic inflationary model, the Multiverse is eternal, even if separate Universes are not eternal. In some Universes, the value of $\varphi$ can be very large, and then they immediately cease to exist as a result of the Big Crunch after the Big Bang. In other Universes, this value can be very close to zero, with the result that they will expand eternally [Bazaluk, 2016].

The modern researches of the standard cosmological model are shown, for example, in the article "A Macroscopic View of the Standard Cosmological Model" by Yury Ignat'ev et al. [Ignat'ev et al., 2018] or "A Cosmological Inflationary Model Using Optimal Control" by Salah Haggag et al. [Haggag et al., 2017].

The standard cosmological model regards exclusively only the structure of the Universe. It does not consider the origin of life and man; therefore, its influence on the educational research is minimized. A number of scientists are trying to explain the origin of man within the boundaries of the standard cosmological model. For example, Ward Blondé in the article "Can an Eternal Life Start from the Minimal Fine-Tuning for Intelligence?" based on the standard cosmological model tries to answer a question "How nature's constants are fine-tuned for the emergence of life?" Blondé answers this question in the following way, "First, universes in the multiverse acquire an unlimited amount of additional finetuning for intelligent life over the course of many universe generations. Such additional fine-tuning may consist of travelling between universes and an afterlife on a distant planet. Second, evolutionary conservation in the evolution of universes in the multiverse provides a declaration why we observe a universe that roughly has the minimal fine-tuning to support intelligent life" [Blondé, 2016: 26]. 


\section{The theory "Evolving Matter"}

In 2000, Oleg Bazaluk proposed a new understanding of the evolution of our Universe, which he represented in the model "Evolving Matter". Subsequently, he improved the argumentation of his theory continuously [Bazaluk, 2009]. The final version of the theory "Evolving Matter" is presented in the monograph "The Theory of Evolution: From a Space Vacuum to Neural Ensembles and Moving Forward" [Bazaluk, 2016]. In the theory "Evolving Matter" the standard cosmological model is not denied. Bazaluk tries to unite the Standard cosmological model, the synthetic theory of evolution and the theory of noogenesis, i.e. the evolution of the Universe, life and mind.

In the theory "Evolving Matter," the author postulated two basic provisions:

1. Evolution is complication of the structure of matter, the types of interaction and the environments, though in the unity and struggle of opposites. Speaking about evolution as complication of the Universe, the author means complication of three components of physical reality: 1) the structure of matter; 2) the types of interaction (relations) between the structures of matter; 3 ) the environments, in which complication of these structures and interactions are carried out, and which, to a varying degree, determine environmental characteristics [Bazaluk, 2016: 35].

2. The complication of any state of matter is based on three factors and two causes of evolution. To the factors of evolution as the complication, Bazaluk assigns: a) Continuity of self-complication of the structure, the types of interaction and the environments of any state of matter, supplemented by blocks of continuous self-complication and the principle of dominance of continuous block self-complication. b) Nonlinear complication of the structure, the types of interaction and the environments of any state of matter, which is added by the factors: hierarchical nonlinear complication and direction of nonlinear hierarchical complication. c) Isolation of complication. Bazaluk attributes the causes of evolution as complication to: a) Active principle, which is inherently the basis for the initial elements of any state of matter and forms self-complication. b) Natural selection as the impact of the external environment. Interaction of the active internal principle of any state of matter with natural selection as the impact of the external environment forms a regulatory compromise [Bazaluk, 2016: 136].

On the basis of the proposed postulates, Bazaluk systematized the accumulated knowledge about the evolution of the Universe, biological life and human and came to the conclusions [Bazaluk, 2016: 129-130]:

1. Complications of the structure of matter, the types of interaction and the environments in our Universe have been carried out by hotbeds, continuously and nonlinearly, over proximately 13.7 billion years. Complication of the structure and functions of the Universe happens under the influence of the same (Universal) factors and causes of evolution. Thus, in the course of evolution, complication of the very factors and causes of evolution themselves happened, which led to the formation and development of the nth number of states of matter.

2. Each state of matter is a new level of complication of the structure of matter, the types of interaction and the environments. In consequence of the complication of each new state of matter comes the formation of invariant hierarchies, providing fixation of the state of matter in the structure of the Universe and its co-evolution with other states of matter. Each new state of matter brings new opportunities for the organization of the circulation of substances, energy and information, as well as ways of moving in space. 
3. Studying the modern scientific and philosophical theories of evolution, Bazaluk discovered the unity of micro and macroevolutionary processes. The basis of this unity is the variability of the factors and causes of evolution.

4. During the work on the evolution models of three states of matter that are known to modern science: Inert Matter, Living Matter and Intelligent Matter, the author discovered and considered the so-called "transition" states of matter. Bazaluk defined them by the terms "Bioinert" and "BioIntelligent" matter.

5. On the example of the Solar System, only one sequence of complication of the structure of the Universe can be seen: Inert Matter $\rightarrow$ Living Matter $\rightarrow$ Intelligent Matter, or alternatively, taking into account "transition" states of matter: Inert Matter $\rightarrow$ Bioinert Matter $\rightarrow$ Living Matter $\rightarrow$ BioIntelligent Matter $\rightarrow$ Intelligent Matter. The modern understanding of evolution and co-evolution of these states of matter is considered in the standard model of the Universe, the synthetic theory of evolution and the concepts of noogenesis.

In the theory "Evolving Matter" the place of Man in scales of the Earth and the Universe as well as prospects of his development are considered. The theory has a direct impact on the educational research, up to the creation of a new theory of education [Bazaluk, 2017a].

\section{The hypothesis of the Mental Universe}

In 2005, Richard Henry gave a strong argument for the lack of an objective physical world that exists independently of being observed. According to Henry, the physical world is contextual: its measurable physical properties do not exist before being observed [Henry, 2005]. Contextuality is a formidable challenge to the viability of realism, especially if we consider that contextuality is predicted by quantum mechanics, and it is not a consequence of philosophical reflections.

The hypothesis of the Mental Universe, suggested by Richard Henry, is confirmed by the studies of Bernardo Kastrup. By combining a modern formulation of the ontology of idealism with the relational interpretation of quantum mechanics, Kastrup proves the main theses of the hypothesis, as well as he considers the key philosophical qualms of the relational interpretation [Kastrup, 2017]. According Kastrup, in a mental universe observation necessarily boils down to perceptual experience and the physical properties of the world exist only insofar as they are perceptually experienced. There is no ontological ground outside mind where these properties could otherwise reside before being represented in mind [Kastrup, 2017: 33]. As a result of work on the hypothesis of the Mental Universe, Kastrup has come to the conclusion that our society stands on the border of a paradigm shift: the materialist worldview gives way to a new form of idealism. He writes: "Idealism can not only accommodate all anomalies amassed to date, but also make sense of all other relevant empirical facts. It is a more parsimonious, empirically robust and explanatorily powerful worldview than materialism" [Kastrup, 2018: 49-50].

The basis of the paradigm of the Mental Universe Kastrup sees in the universal mind, which "is nature's sole fundamental entity, everything else being reducible to excitations of universal mind" [Kastrup, 2018: 49]. The new paradigm should accommodate the kinship and continuity between mind and world. "In other worlds, materialism will be replaced by a form of idealism: the view that a transpersonal mind is the sole fundamental aspect of reality, everything else being reduced to excitations of this mind" [Kastrup, 2018: 47].

Marvin E. Kirsh writes about the impossibility of considering many modern phenomena by rational methods in his article "What in the World is Universe? : A Prime Example" [Kirsh, 
2015; Kirsh, 2016]. However, in order to avoid emerging paradoxes, he suggests another way out: to consider a world constituted of shapes. "Shapes introduced include the three dimensional egg shape, the triangle, pentagon, cube, the helix of DNA, the screw shaped void in the crystal used to investigate Planks constant" [Kirsh, 2016: 115].

The hypothesis of the Mental Universe has a direct impact on the educational research and sets rethinking the role of rational cognition methods that dominate in modern education.

\section{Impact of Cosmological Paradigms on Educational Research}

We reviewed briefly three current ideas about the Universe. In fact, there are many more. We picked up cosmological paradigms in such a way as to emphasize the obvious trend. The authors completely agree with the conclusions reached by Bernardo Kastrup [Kastrup, 2018]. Indeed, our society passes to the new dominant cosmological paradigm, according to which to talk about anthropocentrism, as it was said about geo- and heliocentrism at the time, is incorrectly. The society returns to the Idea of Universe Soul, which is rooted in the philosophy of Antiquity. The history of the Idea of Universe Soul is considered in the article of Igor Goian and Gennadii Aliaiev [Goian \& Aliaiev, 2015]. Rationalism and the values of the Age of Enlightenment, lose their relevance, because Man perceives the Universe depending on the perfection of his brain. This perception is objective and it does not mean at all that outside of it, the Universe is different and it lives its own life. The change of cosmological paradigm has a direct impact on the educational research. Scientific knowledge, like rational thinking, is no longer able to disclose completely the true place of man in the cosmos. The usual technologies of education are replaced by new ones, which try to convey to students the meanings of Intelligent Matter, or the Universe Soul, or the Mental Universe. A new philosophy of education is being formed, which takes into account the tendency of changing the cosmological paradigm and, accordingly, understanding the place of Man in the Universe. In the article "The Theory of Education: "Those Who Transform the Universe"" Oleg Bazaluk announced a new theory of education, built on the basis of contemporary cosmological paradigms [Bazaluk, 2017a]. Continuing his early studies, he insists on the need to build an educational research taking into account the discoveries in neurosciences and cognitive psychology [Bazaluk \& Blazhevych, 2015].

Educational research acquires an obvious structure, which is based on the features of the human brain development in ontogenesis. Moreover, the brain is considered not only and not so much as a neurobiological structure, but as a neural organization involved in the cosmic processes. The hypothesis of the Intelligent Matter and the Mental Universe set rethinking the emphasis in the educational research. The subject of research is the neural structure of the brain and the features of its impact in ontogeny. The object of the researches is the involvement of the neural structure in the cosmic processes and the features of its development under the influence of the cosmos. Quite differently, the scale of man is seen in the educational research. Contemporary cosmological paradigms force one to view man in the educational research beyond the common framework of society. They urge to explore man in the scales of the space, as the planetary-cosmic force, which due to the natural development of brain, with each generation becomes more and more independent and selfsufficient. Knowing the world and improving technology, man becomes part of the cosmic processes that influence the development of the Universe. 


\section{Conclusions}

In the article "Homo Economicus as the Basis of "Asgardia" Nation State in Space: Perspective of Educational Technologies" Roman Oleksenko and Lidia Fedorova write about the creation of the "the Asgardia" nation state in space on the basis of new interpretations of Homo economicus as a cultural ideal for educational technologies [Oleksenko \& Fedorova, 2017]. In the light of current trends in the educational research, their ideas can no longer be perceived as utopias. Contemporary cosmological paradigms push educational research to overcome the limits of consideration of man on the scale of society. Man is more than a society; man is even greater than the planetary force. In the theory "Evolving Matter" and the Mental Universe, man is seen as a cosmic phenomenon, as the Intelligent Matter. In order, the identity of man corresponds to the Intelligent Matter not only the subject and the object of the educational research should be rethought, the educational technologies and understanding of the educational research in human life should be changed. Man as the Intelligent Matter should have the entirely different competencies than those ones he is provided with the modern education. Man as Intelligent Matter will have to solve problems going far beyond the current concepts. For example, research and exploration of the Moon's gas deposits, which were described as an obvious fact by Evgeniy Slyuta [Slyuta, 2017]. Finally, for man like Intelligent Matter, education is not a service, which can be purchased for money, like any material thing on the market. The Platonic senses, as a way of life, should be given back to education. Only in this case, the educational research will be able to convey the meanings of the modern cosmological paradigms to younger generations, namely, the involvement of man in the cosmic processes and consideration of man as the cosmic force.

\section{References}

Bazaluk, Oleg. The Theory of Evolution: From a Space Vacuum to Neural Ensembles and Moving Forward. Cambridge Scholars Publishing, 2016. — 170 p.

Bazaluk, Oleg. The Strategies of Systematization of the Theories of Education. The Main Meaning and Features of the Theories of Education of Plato's and Isocrates' Lines. Future Human Image. Volume 7, 2017: 11-27.

Bazaluk, Oleg. The Theory of Education: "Those Who Transform the Universe" (New Book Announcement). Future Human Image, Volume 8, 2017a: 9-13.

Bazaluk, Oleg. The Existence of the World: Modern Model "Evolving Matter". Philosophy and Cosmology 2009. Poltava, 2009: 3-37.

Bazaluk, Oleg, and Tamara Blazhevych. Modern Basics of the Philosophy of Education. Future Human Image. 2 (5), 2015: 93-100.

Blondé, Ward. Can an Eternal Life Start From the Minimal Fine-Tuning for Intelligence? Philosophy and Cosmology. Volume 17, 2016: 26-38.

Goian, Igor and Gennadii Aliaiev. The Idea of Universe Soul in Empirical Metaphysics of the 19th century (Gustav Theodor Fechner). Philosophy and Cosmology. Volume 14, 2015: 256-271.

Ignat'ev, Yury, D. Yu. Ignatyev, and A. R. Samigullina. A Macroscopic View of the Standard Cosmological Model. Gravitation and Cosmology. Volume 24, Issue 2, 2018: 148 153. DOI: $10.1134 / \mathrm{S} 0202289318020081$

Haggag, Salah, Fatma Desokey and Moutaz Ramadan. A Cosmological Inflationary Model Using Optimal Control. Gravitation and Cosmology. Volume 23, Issue 3, 2017: 236239. 
Henry, Richard C. The Mental Universe. Nature 436, 29, 2005. DOI: 10.1038/436029a

Kastrup, Bernardo. Making Sense of the Mental Universe. Philosophy and Cosmology. Volume 19, 2017: 33-49.

Kastrup, Bernardo. The Next Paradigm. Future Human Image, Volume 9, 2018:41-51. DOI: $10.29202 / \mathrm{fhi} / 9 / 4$

Kirsh, Marvin E. The Spaces in the Looking Glass: Stilling the Frame/Framing the Still. Philosophy and Cosmology. Volume 15, 2015: 62-83.

Kirsh, Marvin E. What in the World is Universe? : A Prime Example. Philosophy and Cosmology. Volume 17, 2016: 106-117.

Oleksenko, Roman and Lidiia Fedorova. Homo Economicus as the Basis of "Asgardia" Nation State in Space: Perspective of Educational Technologies. Future Human Image, Volume 7, 2017: 113-119.

Slyuta, Evgeniy. Problems of Research and Mining of Gas Deposits on the Moon. Mining of Mineral Deposits. 11 (4), 2017:117-125. https://doi.org/10.15407/mining11.04.117 